\title{
Acute High-Intensity Exercise Improves Mood during the Second Trimester of Pregnancy
}

\author{
K Keen ${ }^{1}$, J Maples ${ }^{1}$, B Cooley ${ }^{1}$, A Olenick ${ }^{1}$, M Blankenship ${ }^{2}$, D Hoover ${ }^{3}$ and R Tinius ${ }^{1 *}$ \\ ${ }^{1}$ School of Kinesiology, Recreation, and Sport, Western Kentucky University, Bowling Green, KY, USA \\ ${ }^{2}$ School of Nursing, Western Kentucky University, Bowling Green, $K Y$, USA \\ ${ }^{3}$ Department of Physical Therapy, Western Kentucky University, Bowling Green, KY, USA
}

*Corresponding author: Rachel A Tinius, PhD, ACSM-EP-C, Recreation and Sport, School of Kinesiology, Recreation, and Sport, Western Kentucky University, 1906 College Heights Blvd, \#11089, Bowling Green, KY 42101, USA, Tel: 270-7455026,E-mail: rachel.tinius@wku.edu

\begin{abstract}
Purpose: High-intensity exercise is safe and effective for low-risk pregnant women and their developing babies; however, little is known about the psychological effect of an acute bout of high-intensity exercise among pregnant women. The goal of this study is to examine maternal mood in response to an acute bout of high-intensity exercise.

Methods: Twenty-six pregnant women (18-24 wks gestational age) completed the BRUMS-32 Profile of Mood Survey and Subjective Exercise Experience Scale (SEES). Surveys were administered before and after an acute bout of high-intensity treadmill exercise. Data were entered into SPSS for statistical analysis.

Results: According to the BRUMS-32 Profile of Mood Survey, Total Mood Distress index scores among pregnant women improved significantly after the high-intensity exercise session (pre: $-23.2 \pm 11.8$ vs. post: $-29.0 \pm 10.3, p=$ $0.011)$. When examined as individual categories, pregnant women reported an increase in "Vigor" ( $9.7 \pm 3.1$ vs. $12.3 \pm$ $2.7, p<0.001)$, a decrease in "Tension" $(2.3 \pm 2.8$ vs. $0.6 \pm$ $1.0, p=0.004)$, and a decrease in "Anger" (0.7 \pm 1.4 vs. 0.1 $\pm 0.4, p=0.049$ ) [pre vs. post-exercise] on the BRUMS-32 Survey after high-intensity exercise. In response to the Subjective Exercise Experience Scale, pregnant women's scores for the general category "Positive Well-Being" increased post-exercise (20.8 \pm 3.7 vs. $22.6 \pm 3.9, p=0.015)$, and individual scores for "Strong" increased ( $4.5 \pm 1.1 \mathrm{vs}$. $5.2 \pm 1.3, p=0.003)$ and "Discouraged" decreased (1.6 \pm 0.9 vs. $1.2 \pm 0.5, p=0.03$ ) post-exercise.

Conclusion: High-intensity exercise during pregnancy resulted in improvements in mood; thus, high-intensity exercise may be an effective option for prescribing exercise in pregnant women.
\end{abstract}

\section{Keywords}

High-Intensity exercise, Pregnant women, Mood

\section{Introduction}

Physical activity positively impacts maternal and fetal physiological health [1]. Despite the proven physiological advantages, most pregnant women still do not meet minimal exercise recommendations throughout their pregnancy [2]. In the past, pregnant women were advised to reduce their level of physical activity, particularly high-intensity physical activity, due to increased safety concerns for the pregnant woman and her unborn baby [3]. In fact, 20-years-ago, the American College of Obstetricians and Gynecologists (ACOG) recommended that heart rate during exercise should not exceed 140 beats per minute and that vigorous activity should last no longer than 15 minutes [4]. However, recent research suggests that high-intensity exercise is safe and effective for low-risk pregnant women and their developing babies [5], and updated ACOG guidelines reflect these changes [1]. The intensity of exercise is a key component influencing the extent to which exercise training will lead to various adaptations (e.g. improved aerobic capacity, muscular strength). While there are no specific guidelines prescribing duration and intensity to achieve optimal health benefits from high-intensity exercise, it is understood that as exercise intensity increases, duration can concomitantly decrease while providing positive adaptations [6]. Given

Citation: Keen K, Maples J, Cooley B, Olenick A, Blankenship M, et al. (2017) Acute High-Intensity Exercise Improves Mood during the Second Trimester of Pregnancy. Int J Womens Health Wellness 3:053. doi.org/10.23937/2474-1353/1510053

Received: March 11, 2017: Accepted: May 27, 2017: Published: May 29, 2017

Copyright: (C) 2017 Keen K, et al. This is an open-access article distributed under the terms of the Creative Commons Attribution License, which permits unrestricted use, distribution, and reproduction

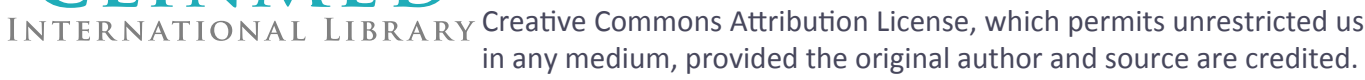


this rational, high-intensity exercise among low-risk pregnant women may be a time-efficient way to improve overall health.

While there is evidence that high-intensity exercise is safe and effective for improving health in low-risk pregnant women [5], little is known about the psychological effect of high-intensity exercise during pregnancy. Because pregnancy can be a very stressful time in a woman's life and depression during and after pregnancy is common [7], understanding the impact of exercise on mood states during pregnancy is important. In addition, stress and anxiety during pregnancy can contribute to postpartum depression - a serious health concern for new mothers [8]. Previous studies have found that longterm physical activity interventions/programs improve mood during pregnancy [9]. Additionally, previous research has shown that acute high-intensity exercise improves mood in non-gravid women [10]; however, no study has investigated the psychological effect of an acute bout of high-intensity exercise among pregnant women. Therefore, the purpose of this study was to examine maternal mood in response to an acute bout of high-intensity exercise. Identifying improvements in mood as a benefit of acute exercise during pregnancy may serve as an additional incentive for pregnant women participate in regular physical activity.

\section{Materials and Methods}

\section{Participants}

Twenty-six low-risk women with uncomplicated pregnancies participated in the study between 18 and 24 weeks gestation. (This gestation age was chosen due to the greatly reduced risk of miscarrying, less need for fetal monitoring, as well as a critical time point in pregnancy in which physicians prescribe exercise). All study procedures were approved by the Western Kentucky University Institutional Review Board (IRB 16-226). Inclusion criteria for the present study included: 1) Pre-Pregnancy BMI between 18 and $25 \mathrm{~kg} / \mathrm{m}^{2}$, 2) Confirmed singleton viable pregnancy with no identified fetal abnormalities (as determined by routine standard of care ultrasonography), 3) Physician clearance for participation in all study procedures, 4) Age 18-44 years, and 5) Exercise regularly and accustomed to regular high-intensity exercise.
Exclusion criteria included: 1) Restrictive lung disease, 2) Incompetent cervix/cerclage, 3) Multiple gestation at risk for premature labor, 4) Persistent $2^{\text {nd }}$ trimester bleeding, 5) Ruptured membranes, 6) Preeclampsia/ pregnancy-induced hypertension, 7) Hemodynamically-significant heart disease, 8) Inability to provide voluntary informed consent, 9) Currently using illegal drugs (e.g. cocaine, methamphetamine, opiates, etc.), and 10) Current smoker.

\section{Study procedures}

Patients reported to the lab after consuming a normal breakfast or lunch. Vitals were obtained upon arrival and any contraindications to the exercise protocol were assessed. Before the acute bout of exercise, patients were given the BRUMS-32 Profile of Mood Survey and the Subjective Exercise Experience Scale (SEES) to assess their mood. Both scales have been validated and used before and after exercise bouts to assess mood [11-13]. The BRUMS-32 Profile of Mood Survey measures eight identifiable affective states through a 32-item self-report inventory with respondents rating a list of adjectives on a 5-point Likert scale ranging from 0 (not at all) to 4 (extremely), based on subjective feelings. Scores from the eight mood state subscales (anger, tension, depression, vigor, fatigue, happy, confusion, and calmness) can also be calculated into a Total Mood Distress (TMD) index. The TMD index is calculated by taking the sum of tension, depression, anger, fatigue, and confusion, and then subtracting the sum of vigor, happy, and calmness (thus, large positive scores represent high levels of distress, and large negative scores represent no distress/good mood state). The Subjective Exercise Experience Scale (SEES) is a brief, 12-item scale assessing three general categories of subjective responses to exercise including positive well-being, psychological distress, and fatigue [13].

The acute bout of exercise followed the Bruce protocol, where treadmill grade and speed increase incrementally until maximal exercise effort is achieved. This protocol allowed the participants time to adjust to each new work rate with 3-minute stages. Heart rate and rating of perceived exertion were monitored throughout the test. On average, the test took 8-12 minutes with the last 2-4 minutes considered high-intensity. Following a 5-minute cool down on the treadmill, the BRUMS-32

\begin{tabular}{|c|c|c|c|c|}
\hline $\begin{array}{c}\text { Prior to study } \\
\text { visit }\end{array}$ & $\begin{array}{c}\text { Upon Arrival to } \\
\text { Lab }\end{array}$ & $\begin{array}{c}\text { Pre Mood } \\
\text { Assessment }\end{array}$ & $\begin{array}{r}\text { High-Intensity } \\
\text { Exercise Bout }\end{array}$ & $\begin{array}{c}\text { Post Mood } \\
\text { Assessment }\end{array}$ \\
\hline - Physican release & $\begin{array}{l}\text { - Infomed Consent } \\
\text { - Vitals and } \\
\text { Anthropometrics }\end{array}$ & $\begin{array}{l}\cdot \text { BRUMS-32 } \\
\text { - SEES }\end{array}$ & $\begin{array}{l}\text { - Treadmill for 8- } \\
15 \text { Minutes } \\
\text { - Maximal Effort } \\
\text { - Bruce Protocol }\end{array}$ & $\begin{array}{l}\text { - } 15 \text {-min Post } \\
\text { Exercise } \\
\text { - } \text { BRUMS-32 } \\
\text { - SEES }\end{array}$ \\
\hline
\end{tabular}

Figure 1: Study protocol. 
Profile of Mood Survey and the Subjective Exercise Experience Scale (SEES) were administered once again (post-exercise). The study protocol is shown in Figure 1.

\section{Statistical analysis}

Paired Samples T-Tests were used to compare pre-exercise scores with post-exercise scores for both surveys. A p-value less than 0.05 were considered statistically significant. All statistical analysis were conducted with SPSS (Version 24).

\section{Results}

Participants were relatively young (mean age was $30.3 \pm 4.0$ years) and had a healthy weight status (mean pre-pregnancy body mass index was $23.1 \pm 3.5 \mathrm{~kg} / \mathrm{m}^{2}$ ) (see Table 1). The majority of participants were Caucasian (96.2\%), had a college education (96.2\%), and nearly all of them exercised at least one time per week (96.2\%). Exercise characteristics demonstrate that, at least a portion, of the exercise bout was indeed high-intensity as heart rate reached $88.4 \%$ of age-predicted maximal heart rate and participants reported an average maximal perceived rating of exertion response of $17.7 \pm 1.5$ on the Borg scale (see Table 2). According to the American College of Sports Medicine, physical ac-

Table 1: Participant demographic characteristics $(n=26)$.

\begin{tabular}{|c|c|}
\hline & $\begin{array}{l}\text { Mean } \pm \text { SD or \# of } \\
\text { women (\%) }\end{array}$ \\
\hline Age (years) & $30.3 \pm 4.0$ \\
\hline Pre-pregnancy BMI (kg/m²) & $23.1 \pm 3.5$ \\
\hline Pregnancy weight (kg) & $63.2 \pm 9.5$ \\
\hline Height (cm) & $165.4 \pm 6.3$ \\
\hline $\begin{array}{l}\text { Resting systolic blood pressure } \\
(\mathrm{mmHg})\end{array}$ & $119.9 \pm 9.8$ \\
\hline $\begin{array}{l}\text { Resting diastolic blood pressure } \\
(\mathrm{mmHg})\end{array}$ & $71.18 \pm 7.2$ \\
\hline \multicolumn{2}{|l|}{ Education } \\
\hline High school diploma & $1(3.8)$ \\
\hline College degree & $14(53.8)$ \\
\hline Post-graduate degree & $11(42.3)$ \\
\hline \multicolumn{2}{|l|}{ Ethnicity } \\
\hline Caucasian & $25(96.2)$ \\
\hline Hispanic & $1(3.8)$ \\
\hline \multicolumn{2}{|l|}{ Physical activity level } \\
\hline None & $1(3.8)$ \\
\hline Once a week & $3(11.5)$ \\
\hline 2-3 times/week & $11(42.3)$ \\
\hline 4-5 times/week & $10(38.5)$ \\
\hline 7 times/week & $1(3.8)$ \\
\hline
\end{tabular}

Table 2: Exercise characteristics.

\begin{tabular}{|l|l|}
\hline & Mean \pm SD \\
\hline $\mathbf{V O}_{2 \max }(\mathrm{ml} / \mathrm{kg} / \mathrm{min})$ & $35.0 \pm 10.2$ \\
\hline $\begin{array}{l}\text { Maximum heart rate }(\mathbf{b p m}) \\
\text { Maximum percent of age-predicted } \\
\text { maximal heart rate (\%) }\end{array}$ & $167.7 \pm 13.7$ \\
\hline $\begin{array}{l}\text { Maximum rating of perceived exertion } \\
\text { (Borg scale, 6-20) }\end{array}$ & $88.4 \pm 7.2$ \\
\hline
\end{tabular}

tivity is classified as "Vigorous" if an individual is as exercising at intensity above $76 \%$ of one's age-predicted maximal heart rate and reports a maximal perceived rating of exertion greater than 14 on the Borg scale [14].

On the BRUMS-32 survey, Total Mood Distress Index improved significantly from pre to post high-intensity exercise (pre: $-23.2 \pm 11.8$ vs. post: $-29.0 \pm 10.3, p=$ 0.011 ). Among the 7 individual categories, three of them were significantly different post-exercise compared to pre-exercise (see Figure 2). Participants reported an increase in "Vigor" ( $9.7 \pm 3.1$ vs. $12.3 \pm 2.7, p<0.001)$, a decrease in "Tension" ( $2.3 \pm 2.8$ vs. $0.6 \pm 1.0, p=0.004)$ and a decrease in "Anger" ( $0.7 \pm 1.4$ vs. $0.1 \pm 0.3, p=$ 0.049 ) after high-intensity exercise. Scores for "Happy", "Fatigue", "Depression", "Confusion," and "Calmness" did not change significantly in response to the bout of exercise.

Three general categories of subjective responses to exercise (i.e. "Positive Well-Being" "Psychological stress," and "Fatigue") were assessed by the 12-item Subjective Exercise Experience Scale (SEES) (see Fig-

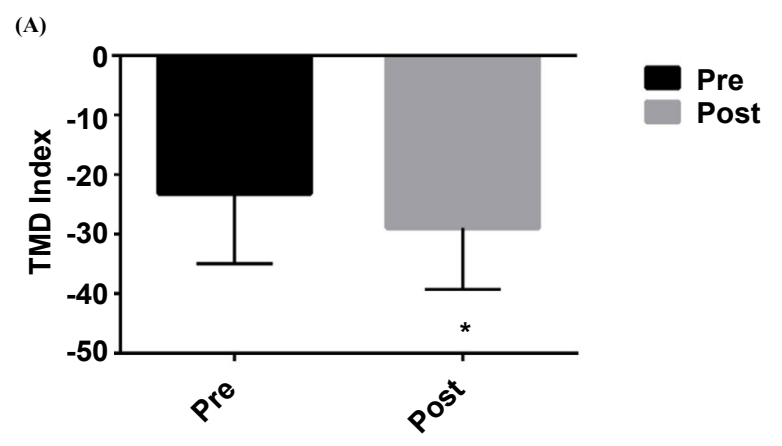

(B)

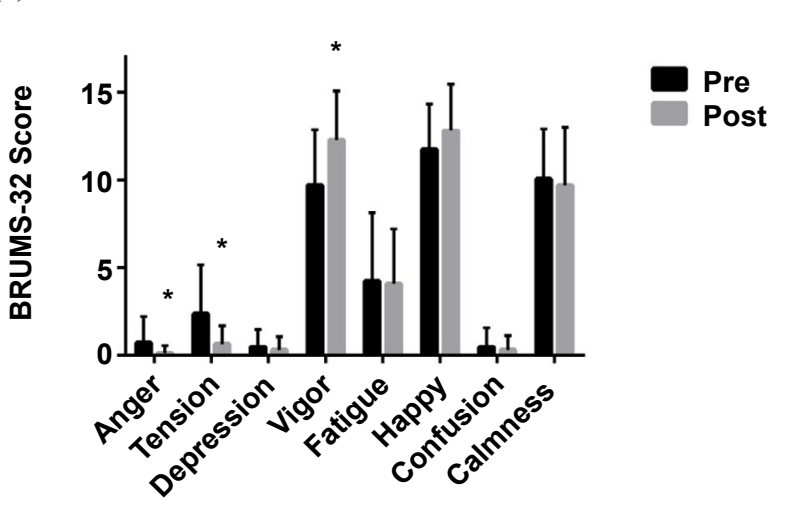

Figure 2: BRUMS-32 scores pre and post high-intensity exercise (A) Total mood distress scores went down following the high-intensity exercise bout - demonstrating an improvement in overall mood following exercise among pregnant women; (B) According to the BRUMS-32 profile of mood survey, anger scores decreased, tension scores decreased, and vigor scores increased in pregnant women after a bout of high-intensity exercise.

* $p<0.05$, a significant increase from pre to post exercise; TMD = Total Mood Distress, large negative scores represent a positive/good mood state. 


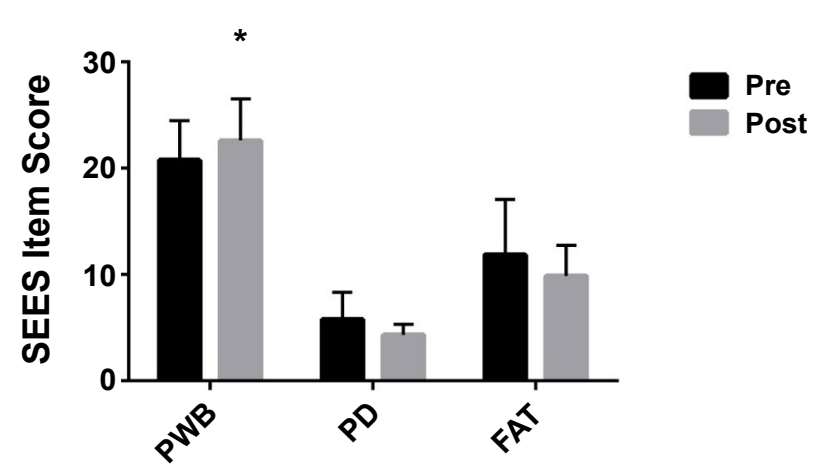

Figure 3: SEES item scores pre and post high-intensity exercise bout. According to the subjective exercise experience scale, positive well-being scores increased in pregnant women after a bout of high-intensity exercise.

* $p<0.05$, a significant increase from pre to post exercise; PWB $=$ Positive Well-Being; $\mathrm{PD}=$ Psychological Distress FAT $=$ Fatigue.

ure 3). The category "Positive Well-Being" was higher post-exercise $(20.8 \pm 3.7$ versus $22.6 \pm 3.9, p=0.015)$, while there were no significant changes in scores for "Psychological stress" and "Fatigue". When the subjective responses to exercise items were examined individually, pregnant women had increased scores for "Strong" ( $4.5 \pm 1.1$ vs. $5.2 \pm 1.3, p=0.003)$ and decreased in scores for "Discouraged" (1.6 \pm 0.9 vs. $1.2 \pm$ $0.5, p=0.03$ ) following the acute bout of high-intensity exercise.

\section{Discussion}

Overall, our findings suggest that an acute bout of high-intensity exercise during pregnancy may elicit improvements in mood during the second trimester of pregnancy. According the BRUMS-32, overall mood was improved following the exercise based on the Total Mood Distress Index. Analyzing specific BRUMS-32 categories, "Anger" and "Tension" scores decreased, while "Vigor" scores increased following acute high-intensity exercise (Figure 2). According to the SEES items/ categories, only the category "Positive well-being" increased post-exercise (Figure 3), and individual scores for "Strong" increased ( $4.5 \pm 1.1$ vs. $5.2 \pm 1.3, p=0.003$ ) and "Discouraged" decreased (1.6 \pm 0.9 vs. $1.2 \pm 0.5, p=$ $0.03)$ post-exercise.

Taken together, this study suggests that high-intensity exercise may be an effective and time-efficient option for prescribing exercise among low-risk, previously active pregnant women. The most recent ACOG recommendations for exercise during pregnancy suggest 20-30 minutes of moderate intensity exercise on most or all days of the week [1], and it is logical to believe that shorter bouts of higher intensity exercise may elicit similar physiological adaptations similar to what is seen in non-gravid populations [15]. By participating in short bouts of moderate-to-high intensity exercise lasting 10-15 minutes day, pregnant women can more easily achieve exercise recommendations and induce valuable physiological adaptations that may improve the health of themselves and their unborn baby.

Anxiety, stress, and depression are very common during pregnancy and postpartum, and the onset of postpartum depression symptoms actually begins during pregnancy [16]. Interestingly, research suggests that there are higher rates of depression, anxiety, and fatigue during pregnancy than following pregnancy; in fact, up to $25 \%$ of pregnant women report symptoms of clinical depression [17-19]. Therefore, methods such as exercise that can improve short and long-term mood status are critical to overall health as psychological health and physical health are closely associated [20]. Anxiety and stress can have negative implications for maternal and neonatal health outcomes [21], so any efforts made to reduce stress and anxiety may also elicit improvements in maternal and neonatal health [22]. A previous study by Gaston and Prapavessis (2013) examining depressive mood scores in pregnant women in response to an exercise program reported a reduction in depressive symptoms after a four-week intervention [23]. This study suggests improvements in mental health can occur in just four weeks. Our study takes this a step further and suggests on a short-term scale, one acute bout of exercise has the potential to improve mood status. This information is extremely encouraging to pregnant women suffering from depression or undergoing mood fluctuations in response to hormonal changes throughout pregnancy. It is also reasonable to believe women who exercise during pregnancy are more likely to continue (once cleared) postpartum [24]; thus, incorporating high-intensity exercise sessions into a daily routine has the potential to lower risk for postpartum depression as well.

In addition to the potential for a plethora of short and long-term health benefits, our study provides rationale for utilizing high-intensity, short duration exercise sessions to overcome the most commonly cited barriers to being physically active during pregnancy: lack of time and lack of energy [25]. This study provides the first evidence supporting the effectiveness of a brief, time-efficient bout of high-intensity exercise on mood scores, including significant improvements in overall mood status, positive well-being, and vigor, in addition to reductions in tension and anger. The exercise test in the current study took 8-12 minutes and elicited improvements in mood. Thus, it is reasonable to suggest even a 10-15 minute workout at a moderate-to-high intensity can elicit these types of mood improvements in pregnant women. Thus, quick workouts can overcome the barrier of lack of time [25]. It is possible beginning to incorporate short workouts into daily routines may actually increase energy levels (i.e. vigor), which may encourage additional physical activity participation and overall improvements in well-being during pregnancy. Therefore, clinicians and health care providers can encourage busy women to set aside even 10-15 minutes to 
exercise so that they can improve their health and the health of their unborn baby.

There are several limitations in the present study. First, the small sample size may have contributed to the lack of significant findings in other mood categories. A larger study with similar objectives is warranted. Next, while the protocol did involve high-intensity/maximal effort exercise, the Bruce protocol is not typical of a self-selected workout routine. Thus, similar studies should be conducted with other high-intensity exercise protocols, and protocols that do not involve a treadmill as many women do not have access to treadmills, to make these results more generalizable. In addition, all mood data was subjective in nature. Future studies should investigate physiological biomarkers linked to mood status as well.

In summary, our study is the first to demonstrate the positive impact of acute, high-intensity exercise on mood status during pregnancy. Low-risk women may want to consider incorporating short bouts of activity in daily routines during pregnancy in order to improve their short and long-term psychological health, which may in turn improve their physical health and the health of their offspring.

\section{Financial Disclosure}

The authors have indicated they have no financial relationships relevant to this article to disclose.

\section{Ethical Statement}

All study procedures were approved by the Western Kentucky University Institutional Review Board (IRB 16226).

\section{Acknowledgements}

FUSE 17-SP213, FUSE 16-SP212, NIH NIGMS IDeA Grant 5P20GM103436.

\section{References}

1. (2015) ACOG Committee Opinion No. 650: Physical Activity and Exercise During Pregnancy and the Postpartum Period. Obstet Gynecol 126: e135-e142.

2. Evenson KR, Wen $F(2010)$ National trends in self-reported physical activity and sedentary behaviors among pregnant women: NHANES 1999-2006. Prev Med 50: 123-128.

3. Hammer RL, Perkins J, Parr R (2000) Exercise During the Childbearing Year. J Perinat Educ 9: 1-14.

4. (1985) American College of Obstetricians and Gynecologists. Exercise during pregnancy and the postnatal period. ACOG Home Exercise Programs, Washington DC, 1-6.

5. Szymanski LM, Satin AJ (2012) Strenuous exercise during pregnancy: is there a limit? Am J Obstet Gynecol 207: 179. e1-179.e6.

6. Dudley GA, Abraham WM, Terjung RL (1982) Influence of exercise intensity and duration on biochemical adaptations in skeletal muscle. J Appl Physiol Respir Environ Exerc Physiol 53: 844-850.

7. Gavin NI, Gaynes BN, Lohr KN, Meltzer-Brody S, Gartlehner G, et al. (2005) Perinatal depression: a systematic review of prevalence and incidence. Obstet Gynecol 106: 1071-1083.

8. Leuner B, Fredericks PJ, Nealer C, Albin-Brooks C (2014) Chronic gestational stress leads to depressive-like behavior and compromises medial prefrontal cortex structure and function during the postpartum period. PLoS One 9: e89912.

9. Taniguchi C, Sato C (2016) Home-based walking during pregnancy affects mood and birth outcomes among sedentary women: A randomized controlled trial. Int J Nurs Pract 22: 420-426.

10. Pronk NP, Crouse SF, Rohack JJ (1995) Maximal exercise and acute mood response in women. Physiol Behav 57: $1-4$.

11. Lane AM, Milton KE, Terry PC (2005) Personality does not influence exercise-induced mood enhancement among female exercisers. J Sports Med Phys Fitness 45: 208-212.

12. Brandt R, Herrero D, Massetti T, Crocetta TB, Guarnieri $R$, et al. (2016) The Brunel Mood Scale Rating in Mental Health for Physically Activity and Apparently Healthy Populations. Scientific Research 8: 125-132.

13. McAuley E, Courneya KS (1994) The Subjective Exercise Experience Scale (SEES): Development and Preliminary Validation. Journal of Sport and Exercise Psychology 16: 163-177.

14. Wolters Kluwer (2014) ACSM's Guidelines for Exercise Testing and Prescription 9th Ed. 2014. J Can Chiropr Assoc 58: 328.

15. Boutcher SH (2011) High-intensity intermittent exercise and fat loss. J Obes 2011: 868305.

16. Stowe ZN, Hostetter AL, Newport DJ (2005) The onset of postpartum depression: Implications for clinical screening in obstetrical and primary care. Am J Obstet Gynecol 192: 522-526.

17. Poudevigne MS, O'Connor PJ (2006) A review of physical activity patterns in pregnant women and their relationship to psychological health. Sports Med 36: 19-38.

18. Field T, Diego M, Hernandez-Reif M, Schanberg S, Kuhn C, et al. (2003) Pregnancy anxiety and comorbid depression and anger: effects on the fetus and neonate. Depress Anxiety 17: 140-151.

19. Gotlib IH, Whiffen VE, Mount JH, Milne K, Cordy NI (1989) Prevalence rates and demographic characteristics associated with depression in pregnancy and the postpartum. J Consult Clin Psychol 57: 269-274.

20. Goldberg D (2010) The detection and treatment of depression in the physically ill. World Psychiatry 9: 16-20.

21. Conde A, Figueiredo B, Tendais I, Teixeira C, Costa R, et al. (2010) Mother's anxiety and depression and associated risk factors during early pregnancy: effects on fetal growth and activity at 20-22 weeks of gestation. J Psychosom Obstet Gynaecol 31: 70-82.

22. Geetha Shivakumar, Anna R Brandon, Peter G Snell, Patricia Santiago-Muñoz, Neysa L Johnson, et al. (2011) Antenatal depression: a rationale for studying exercise. Depress Anxiety 28: 234-242.

23. Gaston A, Prapavessis H (2013) Tired, moody and pregnant? Exercise may be the answer. Psychol Health 28: 1353-1369.

24. Borodulin K, Evenson KR, Herring AH (2009) Physical activity patterns during pregnancy through postpartum. BMC Womens Health 9: 32.

25. Cramp AG, Bray SR (2009) A prospective examination of exercise and barrier self-efficacy to engage in leisure-time physical activity during pregnancy. Ann Behav Med 37: 325-334. 